\section{Efeito do exercício físico aeróbico e de força no perfil lipídico de seus praticantes: uma revisão sistemática}

\section{Effects of aerobic and strength exercise on the lipid profile of its practitioners: a systematic review}

Allan Irwin Leite Bezerra ${ }^{1}$

Hélcio Kanegusuku ${ }^{1}$

Wagner Luiz do Prado ${ }^{2}$

Raphael Mendes Ritti Dias ${ }^{2}$

Crivaldo Gomes Cardoso Júnior ${ }^{3}$

\section{Resumo}

O objetivo deste estudo foi avaliar os efeitos agudos e crônicos dos exercícios aeróbicos e de força no perfil lipídico de seus praticantes. Para tanto, foi conduzida uma revisão sistemática de artigos indexados no PubMed/MedLine, LILACS e SCIELO até agosto de 2011 com estudos aleatorizados, pareados por grupo controle, conduzidos com seres humanos e escritos em português ou inglês. As evidências encontradas advogam em favor do exercício aeróbico, tanto de forma aguda quanto crônica, para aumentar os níveis das lipoproteínas de alta densidade e reduzir os níveis de triglicérides. Os efeitos do exercício de força ainda permanecem por serem estabelecidos pela carência de estudos até o presente momento. Em conclusão, os exercícios aeróbicos permanecem como indicação preferencial para adequação do perfil lipídico de seus praticantes.

\section{Palavras-chave}

Dislipidemias; Exercício; Lipoproteínas.

\begin{abstract}
The aim of this study was to evaluate the acute and chronic effects of aerobic and strength exercise on the lipid profile of its practitioners. For this, we conducted a systematic review of articles indexed in PubMed / MedLine, LILACS and SciELO until August 2011 with randomized, matched control group, conducted with humans and written in Portuguese or English. The evidence found advocates in favor that both, acute and chronic aerobic exercise, increase the levels of high density lipoproteins and reduce the levels of triglycerides. The effects of resistance exercise still remain to be established by the lack of studies to date. In conclusion, aerobic exercises remain as the preferred treatment for adjustment of the lipid profile of its practitioners.
\end{abstract}

\section{Keywords}

Dyslipidemia; Exercise; Lipoprotein.
Rev Bras Ativ Fis Saúde p. 399-411

DOI:

http://dx.doi.org/10.12820/rbafs.v.18n4p399

1 Escola de Educação Física e Esporte. Universidade de São Paulo, SP.

2 Escola Superior de Educação Física. Universidade de Pernambuco, PE.

3 Centro de Educação Física e Esporte, Universidade Estadual de Londrina, PR. 


\section{INTRODUÇÃO}

As doenças arteriais coronarianas (DAC) constituem a principal causa de morte em nosso país dentre os adultos jovens e de meia idade (até 50 anos). Neste contexto, apesar dos marcadores etiológicos desta doença ainda não terem sido plenamente identificados, sabe-se que os distúrbios no metabolismo dos lipídeos e das lipoproteínas são fatores patogênicos comuns para a formação da placa aterosclerótica e aumentam o risco para doenças arteriais coronarianas (DAC) ${ }^{1}$. Em termos epidemiológicos, dados oriundos de um estudo ${ }^{3}$ realizado nas capitais brasileiras e no Distrito Federal com 49.395 indivíduos apontaram que 16,5\% apresentavam níveis alterados no perfil lipídico. Nesse sentido, há de se destacar que a elevação em 1\% nas lipoproteínas de alta densidade (HDL) se associa com redução significante de 3\% nas taxas de mortalidade cardiovascular ${ }^{4}$. Assim, medidas terapêuticas de combate às dislipidemias são necessárias para diminuir a mortalidade cardiovascular, bem como para atenuar o elevado custo social e econômico decorrentes desta doença.

A prática regular do exercício físico tem sido indicada como uma importante estratégia não farmacológica, de baixo custo e de fácil acesso para a prevenção e tratamento das dislipidemias ${ }^{5}$. Este efeito salutar do exercício físico impacta diretamente na redução do risco cardiovascular global ${ }^{6}$. Entretanto, especula-se existir uma relação dose-resposta entre intensidade do exercício físico e o perfil lipídico. No entanto, quando essa problemática foi investigada ${ }^{7}$ não pôde ser constatada a referida relação, o que parece ocorrer pela dificuldade em comparar os diferentes protocolos de exercícios físicos, bem como a população submetida a este exercício.

Ainda assim, a IV Diretriz Brasileira sobre dislipidemias e prevenção da aterosclerose $^{5}$ recomenda a prática regular de exercícios aeróbicos, realizados de três a seis vezes/semana, em sessões de 30 a 60 min com intensidades de $60-80 \%$ da frequência cardíaca máxima (FCmax), bem como a realização do exercício de força em complemento ao exercício aeróbico com sobrecargas de 50\% de uma contração voluntária máxima. Todavia, essas recomendações foram publicadas há algum tempo, e desde então diversos estudos têm sido realizados sobre essa temática. Portanto, é premente a necessidade de uma síntese das evidências disponíveis até o momento acerca do efeito agudo e crônico dos exercícios aeróbicos e de força no perfil das lipoproteínas de seus praticantes, visando contribuir para a estruturação dos programas de exercícios físicos como medida terapêutica das dislipidemias, bem como no auxílio à comunidade científica na identificação das características dos exercícios físicos que carecem de investigação.

Dessa forma, o objetivo deste estudo foi reunir um corpo de evidências, por meio de uma revisão sistemática, buscando avaliar os efeitos dos exercícios aeróbicos e de força realizados tanto de forma aguda quanto crônica, nas dislipidemias.

\section{MÉTODOS}

Foi realizada uma revisão sistemática dos artigos científicos publicados em periódicos indexados, nas bases de dados eletrônicas PubMed/MedLine (National Library of Medicine), LILACS (Literatura Latino-Americana e do Caribe em Ciências da Saúde) e SCIELO (Scientific Eletronic Library Online) entre o período de 1980 até agosto de 2011. Como critérios de inclusão, os estudos deveriam ser aleatorizados, pareados por grupo controle, conduzidos em seres humanos e escritos em português ou inglês. 
Todas as etapas do processo de revisão (busca eletrônica nas bases de dados, seleção, avaliação e extração de informações dos estudos potenciais) foram realizadas por dois pesquisadores independentes. Ademais, os resultados obtidos foram gerenciados e comparados por um terceiro pesquisador que, além de contribuir para sanar uma eventual divergência, também foi responsável por comparar a concordância entre os pesquisadores.

Inicialmente, foram consultadas as bases de dados MeSH - Medical Subject Headings, pelo endereço eletrônico da U. S. National Library of Medical (NLM); e DeCS - Descritores em Ciências da Saúde, pelo endereço eletrônico da Biblioteca Virtual em Saúde (BVS) para a identificação dos descritores/termos em português e inglês, respectivamente: colesterol/cholesterol; colesterol, HDL/cholesterol HDL; colesterol, LDL/cholesterol LDL; colesterol, VLDL/cholesterol VLDL; lipoproteínas/lipoprotein; triglicérides/triglycerides; exercício/exercise; exercício resistido/resistance exercise; e, condicionamento físico/physical fitness. Estes descritores foram agrupados em duas categorias nomeadas como lípides e exercícios e, posteriormente, foram agrupados em uma única categoria de forma combinada.

Na sequência, foram excluídos os artigos em duplicata e fez-se a leitura dos resumos. Somente foram incluídos os artigos que utilizaram o exercício físico como forma de intervenção e que apresentavam desfecho nas lipoproteínas. $\mathrm{Na}$ etapa subsequente foi realizada a leitura dos artigos na íntegra e conduzida a extração dos dados referentes à população estudada, ao protocolo de exercício físico realizado e aos desfechos clínicos pertinentes.

Para a identificação dos indicadores de validade interna dos estudos selecionados foi empregada a escala PEDro ${ }^{8}$.

\section{RESULTADOS}

Ao todo foram identificados 10301 artigos, porém apenas 84 destes foram incluídos pela leitura dos títulos. Destes, 34 foram excluídos por estarem duplicados em duas ou mais bases de dados, 16 por não apresentarem o exercício como forma de intervenção e quatro por não terem grupo controle. Ao final, 30 estudos foram incluídos, dos quais 27 foram publicados em periódicos indexados no PubMed/ MedLine, um no LILACS e dois no SCIELO. O fluxograma de seleção dos estudos está apresentado na figura 1.

Dentre os estudos incluídos, 23 avaliaram o efeito do treinamento aeróbico, três o efeito do treinamento de força e cinco o efeito agudo do exercício aeróbico. Nenhum estudo avaliou o efeito agudo do exercício de força. A análise da escala de PEDro revelou pontuação média de 6,5 variando de 4-9 pontos.

Todos os estudos que avaliaram o efeito agudo do exercício aeróbico sobre o perfil lipídico foram realizados com população de normolipidêmicos, e as características estão apresentadas na tabela 1 . A variação etária foi de 18-47 anos e nenhum estudo relatou o uso de medicamentos hipolipemiantes. Em dois estudos, o exercício físico foi realizado em esteira, e nos demais em cicloergômetro. A intensidade do exercício variou de $40-75 \%$ do consumo máximo de oxigênio $\left(\mathrm{VO}_{2} \max \right)$ e a duração das sessões variou de 30-90 min.

Redução nos níveis de colesterol total foi constatada em apenas um estudo, enquanto nenhum estudo demonstrou diminuição nos níveis de LDL. Já, os níveis de TG reduziram em todos os estudos em que ele foi avaliado $(\mathrm{N}=4)$ e o HDL aumentou significantemente em dois deles. A caracterização do exercício aeróbico 


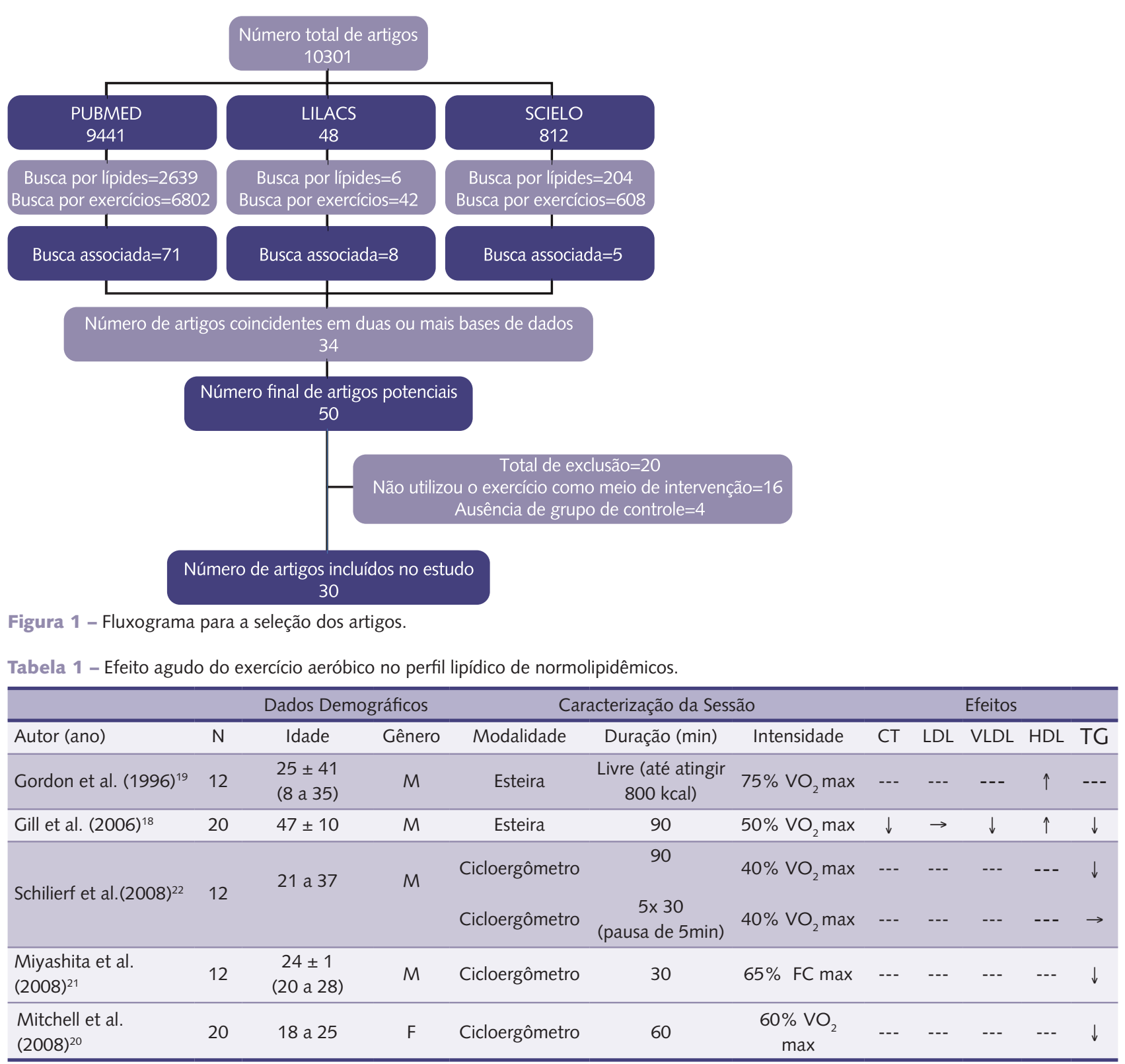

$\mathrm{CT}=$ colesterol total; $\mathrm{LDL}=$ lipoproteína de baixa densidade; $\mathrm{VLDL}=$ lipoproteína de densidade muito baixa; $\mathrm{HDL}=$ lipoproteína de alta densidade; $\mathrm{TG}=$ triglicérides; $M=$ masculino; $F=$ feminino; $\mathrm{VO}_{2} \max =$ Consumo máximo de oxigênio; $F C$ max= frequência cardíaca máxima; $\downarrow=$ reduz: $\rightarrow=$ mantém; $\uparrow=$ aumenta; $---=$ não relatado.

para a redução aguda do colesterol total foi: caminhada/corrida em $50 \% \mathrm{VO}_{2}$ max com duração de 90 min. Para a diminuição aguda dos TG foi encontrado tanto com exercícios de caminhada quanto com aqueles realizados em cicloergômetro, com intensidades de 40-60\% $\mathrm{VO}_{2}$ max e durações de 30-90 min. Para o aumento agudo da HDL, os exercícios de caminhada em esteira foram mais eficazes do que pedalar no cicloergômetro, e a intensidade foi de 50-75\% $\mathrm{VO}_{2}$ max com duração de $90 \mathrm{~min}$ ou até que fosse atingido um total de $800 \mathrm{kcal}$.

As características gerais dos estudos que analisaram os efeitos do treinamento aeróbico em sujeitos normolipidêmicos $(\mathrm{N}=19)$ e dislipidêmicos $(\mathrm{N}=4)$ estão apresentadas nas tabelas 2 e 3 , respectivamente.

Dos 23 estudos selecionados, 19 avaliaram o efeito crônico do exercício aeróbico em indivíduos normolipidêmicos e quatro em dislipidêmicos. Além disso, em 
Tabela 2 - Efeito crônico do exercício aeróbico no perfil lipídico de normolipidêmicos.

\begin{tabular}{|c|c|c|c|c|c|c|c|c|c|c|c|c|c|}
\hline & $\mathrm{Dad}$ & os Demo & gráficos & & Caracteriza & ção do Treinar & nento & & & & Desfech & & \\
\hline Autor (ano) & $\mathrm{N}$ & Idade & Gênero & $\begin{array}{l}\text { Exposição } \\
\text { (sem) }\end{array}$ & Modalidade & $\begin{array}{l}\text { Frequência } \\
\text { (vezes/sem) }\end{array}$ & $\begin{array}{c}\text { Duração } \\
\text { (min) }\end{array}$ & Intensidade & $\mathrm{CT}$ & LDL & VLDL & $\mathrm{HDL}$ & TG \\
\hline \multirow{3}{*}{$\begin{array}{l}\text { Alam et al. } \\
(2004)^{23}\end{array}$} & 9 & $60 \pm 3$ & & & $\begin{array}{c}\text { Livre } \\
\text { (supervisionado) }\end{array}$ & & & & $\rightarrow$ & $\rightarrow$ & $\downarrow$ & $\uparrow$ & $\downarrow$ \\
\hline & & & $F / M$ & 24 & & 4 & $20-40$ & $\begin{array}{l}60-85 \% \\
\mathrm{VO}_{2} \text { máx }\end{array}$ & & & & & \\
\hline & 9 & $55 \pm 3$ & & & $\begin{array}{c}\text { Livre (não } \\
\text { supervisionado) }\end{array}$ & & & & $\rightarrow$ & $\rightarrow$ & $\rightarrow$ & $\rightarrow$ & $\rightarrow$ \\
\hline \multirow{3}{*}{$\begin{array}{l}\text { Agren et al. } \\
(1989)^{24}\end{array}$} & 18 & & & & Ciclo & & & & $\rightarrow$ & $\rightarrow$ & $\downarrow$ & $\uparrow \uparrow$ & $\downarrow$ \\
\hline & & $52 \pm 9$ & M & 12 & & 3 & 60 & 80\% FCmáx & & & & & \\
\hline & 19 & & & & Controle & & & & $\rightarrow$ & $\rightarrow$ & $\rightarrow$ & $\uparrow$ & $\rightarrow$ \\
\hline \multirow{3}{*}{$\begin{array}{l}\text { Brown et al. } \\
(2009)^{25}\end{array}$} & 8 & & & & $\begin{array}{l}\text { Esteira, elíptico ou } \\
\text { ciclo }\end{array}$ & --- & 60 & $\begin{array}{l}40-60 \% \\
\mathrm{VO}_{2} \max \end{array}$ & --- & $\rightarrow$ & $\downarrow$ & $\uparrow$ & $\downarrow$ \\
\hline & & 18 a 50 & $\mathrm{~F}$ & 20 a 24 & & & & & & & & & \\
\hline & 12 & & & & Controle & & & & --- & $\rightarrow$ & $\rightarrow$ & $\rightarrow$ & $\rightarrow$ \\
\hline \multirow{4}{*}{$\begin{array}{l}\text { Busby et al. } \\
(1985)^{26}\end{array}$} & 12 & $49 \pm 5$ & $\mathrm{~F}$ & 12 & Caminhada & 3 & 60 & 70-80\% FCmáx & $\uparrow$ & $\rightarrow$ & $\rightarrow$ & $\rightarrow$ & $\rightarrow$ \\
\hline & 8 & $54 \pm 5$ & $\mathrm{~F}$ & 12 & Terapia de grupo & 2 & 120 & --- & $\rightarrow$ & $\rightarrow$ & $\rightarrow$ & $\rightarrow$ & $\rightarrow$ \\
\hline & 12 & $55 \pm 5$ & $\mathrm{~F}$ & 12 & Caminhada + TG & $3+2$ & $60+120$ & 70-80\% FCmáx & $\rightarrow$ & $\rightarrow$ & $\rightarrow$ & $\rightarrow$ & $\rightarrow$ \\
\hline & 12 & $50 \pm 7$ & $\mathrm{~F}$ & 12 & Controle & --- & --- & --- & $\rightarrow$ & $\rightarrow$ & $\rightarrow$ & $\rightarrow$ & $\rightarrow$ \\
\hline \multirow{3}{*}{$\begin{array}{l}\text { Cauley et al. } \\
(1987)^{27}\end{array}$} & 114 & $58 \pm 4$ & & & Caminhada & 2 & --- & 7 milhas/sem & --- & --- & --- & $\rightarrow$ & --- \\
\hline & & & $\mathrm{F}$ & 96 & & & & & & & & & \\
\hline & 115 & $57 \pm 4$ & & & Controle & --- & --- & --- & --- & --- & --- & $\rightarrow$ & --- \\
\hline \multirow{3}{*}{$\begin{array}{l}\text { Cox et al. } \\
(1990)^{28}\end{array}$} & & $37 \pm 1$ & & & Ciclo & 3 & 30 & $\begin{array}{l}60-70 \% \\
\text { Cargamáx }\end{array}$ & $\rightarrow$ & --- & --- & $\rightarrow$ & $\rightarrow$ \\
\hline & 72 & & $M$ & 4 & & & & & & & & & \\
\hline & & $37 \pm 1$ & & & Controle ativo & 3 & 40 & Carga mínima & $\rightarrow$ & --- & --- & $\rightarrow$ & $\rightarrow$ \\
\hline \multirow{3}{*}{$\begin{array}{l}\text { Gavin et al. } \\
(2010)^{15}\end{array}$} & 60 & $54 \pm 7$ & & & Caminhada + Ciclo & 3 & 45 & $75 \%$ FCmax & $\rightarrow$ & $\rightarrow$ & $\rightarrow$ & $\rightarrow$ & $\rightarrow$ \\
\hline & & & F/M & 24 & & & & & & & & & \\
\hline & 63 & $55 \pm 7$ & & & Controle & --- & --- & --- & $\rightarrow$ & $\rightarrow$ & $\rightarrow$ & $\rightarrow$ & $\rightarrow$ \\
\hline $\begin{array}{l}\text { Grandjean et } \\
\text { al. }(1996)^{29}\end{array}$ & 20 & --- & $\mathrm{F}$ & 24 & $\begin{array}{l}\text { Caminhada/ } \\
\text { Corrida/Ciclo }\end{array}$ & 3 & $20-60$ & $\begin{array}{l}60-80 \% \\
\mathrm{VO}_{2} \text { máx }\end{array}$ & $\rightarrow$ & $\rightarrow$ & $\rightarrow$ & $\rightarrow$ & $\rightarrow$ \\
\hline \multirow{3}{*}{$\begin{array}{l}\text { Marti et al. } \\
(1990)^{30}\end{array}$} & 39 & & & & $\begin{array}{l}\text { Corrida e/ou } \\
\text { Caminhada }\end{array}$ & $2-6$ & 120 & $85 \%$ FC do LV1 & $\rightarrow$ & $\downarrow$ & $\downarrow$ & $\uparrow$ & $\rightarrow$ \\
\hline & & $39 \pm 9$ & M & 16 & & & & & & & & & \\
\hline & 22 & & & & Controle & --- & $-\cdots$ & --- & $\rightarrow$ & $\rightarrow$ & $\rightarrow$ & $\rightarrow$ & $\rightarrow$ \\
\hline \multirow{3}{*}{$\begin{array}{l}\text { Moreira et } \\
\text { al. }(2008)^{31}\end{array}$} & 22 & $40 \pm 8$ & F/M & 12 & Ciclo & 3 & $20-60$ & $10 \%<\mathrm{LV} 1$ & $\downarrow$ & --- & --- & --- & $\rightarrow$ \\
\hline & 22 & $40 \pm 8$ & $\mathrm{~F} / \mathrm{M}$ & 12 & Ciclo intermitente & 3 & $20-60$ & $20 \%>$ LV1 & $\rightarrow$ & --- & --- & --- & $\rightarrow$ \\
\hline & 22 & $40 \pm 8$ & $\mathrm{~F} / \mathrm{M}$ & 12 & Controle & --- & --- & --- & $\rightarrow$ & --- & --- & --- & $\rightarrow$ \\
\hline \multirow{4}{*}{$\begin{array}{l}\text { Nieman et } \\
\text { al. }(2002)^{32}\end{array}$} & 21 & 25 a 75 & $\mathrm{~F}$ & 12 & Caminhada & 5 & 45 & 60-80\% FCmáx & $\rightarrow$ & $\rightarrow$ & $\rightarrow$ & $\rightarrow$ & $\rightarrow$ \\
\hline & 22 & 25 a 75 & $\mathrm{~F}$ & 12 & Caminhada + Dieta & 5 & 45 & $<100 \mathrm{bpm}$ & $\downarrow$ & $\downarrow$ & $\rightarrow$ & $\rightarrow$ & $\downarrow$ \\
\hline & 26 & 25 a 75 & $\mathrm{~F}$ & 12 & $\begin{array}{c}\text { Dieta } \\
\text { (alongamento+ } \\
\text { calistenia leve) }\end{array}$ & 4 & 45 & $<100 \mathrm{bpm}$ & $\downarrow$ & $\downarrow$ & $\rightarrow$ & $\rightarrow$ & $\downarrow$ \\
\hline & 22 & 25 a 75 & $F$ & 12 & $\begin{array}{c}\text { Controle } \\
\text { (alongamento+ } \\
\text { calistenia leve) }\end{array}$ & 4 & 45 & $<100$ bpm & $\rightarrow$ & $\rightarrow$ & $\rightarrow$ & $\rightarrow$ & $\rightarrow$ \\
\hline
\end{tabular}




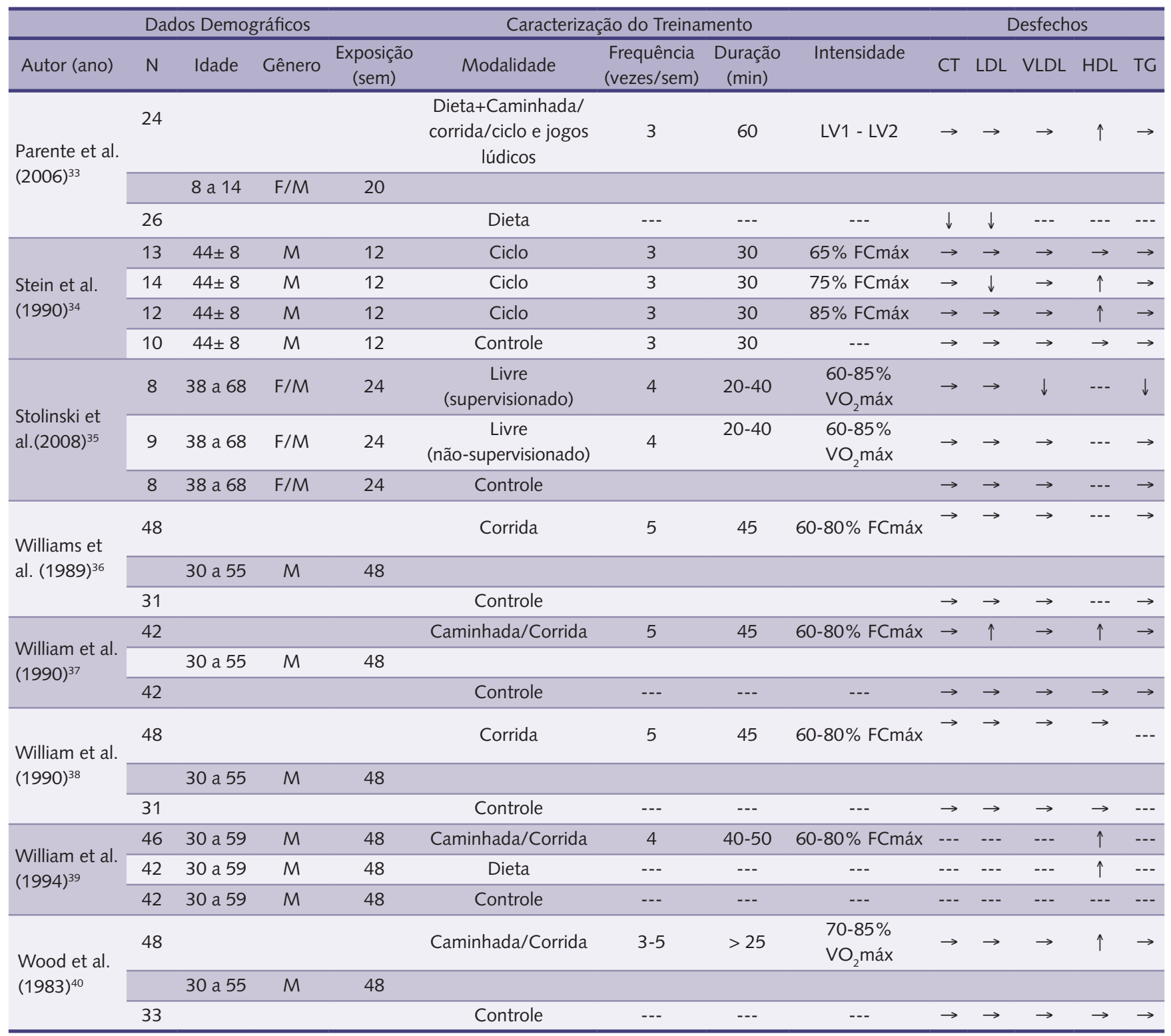

CT: Colesterol Total; LDL: Lipoproteína de baixa densidade; VLDL: Lipoproteína de muito baixa densidade; HDL: lipoproteína de alta densidade; TG: triglicérides; $M=$ masculino; $F=$ feminino; $V O_{2} \max$ : Consumo máximo de oxigênio; FCmax: frequência cardíaca máxima; bpm : batimentos por minuto; LV1: limiar ventilatório 1; LV2: Limiar ventilatório 2 ; $\downarrow$ : reduz: $\rightarrow$ : mantém; $\uparrow:$ aumenta; --- : não relatado.

10 estudos a análise foi realizada somente com o gênero masculino (30-59 anos), sendo um com idosos e um com adolescentes. Em oito foram analisados os dados de ambos os gêneros (8-70 anos) e em cinco a análise foi conduzida somente com o gênero feminino (18-75 anos). O uso concomitante de medicamentos hipolipemiantes (esteróis vegetais, estatinas e fibratos) foi relatado em dois estudos. Já, a realização de dieta hipocalórica concomitante ao treinamento foi relatada em quatro. Em todos os outros estudos, houve orientação para a manutenção dos hábitos alimentares.

O tempo de treinamento físico aeróbico variou de 4-24 meses, com frequência de 3-6 vezes/semana, e intensidade de 40-85\% do consumo pico de oxigênio ( $\mathrm{VO}_{2}$ pico) ou de 60-80\% da frequência cardíaca máxima (FCmax) e com duração de 20-120 min/sessão. As modalidades mais empregadas foram a caminhada e 
Tabela 3 - Efeito crônico do exercício aeróbico no perfil lipídico de dislipidêmicos.

\begin{tabular}{|c|c|c|c|c|c|c|c|c|c|c|c|c|c|}
\hline \multirow[b]{2}{*}{ Autor (ano) } & \multirow[b]{2}{*}{$\mathrm{N}$} & \multicolumn{2}{|c|}{$\begin{array}{c}\text { Dados } \\
\text { Demográficos }\end{array}$} & \multicolumn{5}{|c|}{ Caracterização do Treinamento } & \multicolumn{5}{|c|}{ Desfechos } \\
\hline & & Idade & Gênero & $\begin{array}{l}\text { Exposição } \\
\text { (sem) }\end{array}$ & Modalidade & $\begin{array}{l}\text { Frequência } \\
\text { (vezes/sem) }\end{array}$ & $\begin{array}{c}\text { Duração } \\
\text { (min) }\end{array}$ & Intensidade & CT & LDL & VLDL & $\mathrm{HDL}$ & TG \\
\hline \multirow{4}{*}{$\begin{array}{l}\text { Holme et al. } \\
(2007)^{41}\end{array}$} & 54 & 41 a 50 & $\mathrm{~F} / \mathrm{M}$ & 48 & $\begin{array}{l}\text { Aeróbica/ } \\
\text { Caminhada/Corrida }\end{array}$ & 3 & 60 & $\begin{array}{l}\text { 60-80\% } \\
\text { FCmáx }\end{array}$ & --- & $\rightarrow$ & --- & -- & --- \\
\hline & 67 & 41 a 50 & $\mathrm{~F} / \mathrm{M}$ & 48 & $\begin{array}{l}\text { Dieta + Aeróbica/ } \\
\text { Caminhada/Corrida }\end{array}$ & 3 & 60 & $\begin{array}{l}60-80 \% \\
\text { FCmáx }\end{array}$ & --- & --- & --- & --- & --- \\
\hline & 55 & 41 a 50 & $\mathrm{~F} / \mathrm{M}$ & 48 & Dieta & -- & -- & --- & --- & --- & --- & --- & --- \\
\hline & 43 & 41 a 50 & $\mathrm{~F} / \mathrm{M}$ & 48 & Controle & -- & -- & --- & --- & --- & --- & --- & --- \\
\hline \multirow{3}{*}{$\begin{array}{l}\text { Raz et al. } \\
(1988)^{13}\end{array}$} & 28 & & M & & $\begin{array}{l}\text { Corrida/Corrida }+ \\
\text { Circuito }\end{array}$ & 3 & 50 & $\begin{array}{l}70-85 \% \\
\mathrm{VO}_{2} \text { máx }\end{array}$ & $\rightarrow$ & $\rightarrow$ & --- & $\rightarrow$ & $\downarrow$ \\
\hline & & $25 \pm 1$ & $M$ & 9 & & & & & & & & & \\
\hline & 27 & & & & Controle & --- & --- & --- & $\rightarrow$ & $\rightarrow$ & --- & $\rightarrow$ & $\rightarrow$ \\
\hline \multirow{4}{*}{$\begin{array}{l}\text { Stefanick et } \\
\text { al. }(1998)^{11}\end{array}$} & 49 a 47 & 30 a 64 & $M / F$ & 48 & Caminhada rápida & 3 & --- & $\begin{array}{l}16 \text { milhas/ } \\
\text { sem }\end{array}$ & $\rightarrow$ & $\rightarrow$ & --- & $\rightarrow$ & $\rightarrow$ \\
\hline & 50 a 44 & 30 a 64 & $M / F$ & 48 & $\begin{array}{l}\text { Caminhada rápida } \\
+ \text { Dieta }\end{array}$ & 3 & --- & $\begin{array}{l}16 \text { milhas/ } \\
\text { sem }\end{array}$ & $\downarrow$ & $\downarrow$ & --- & $\rightarrow$ & $\rightarrow$ \\
\hline & 51 a 43 & 30 a 64 & $M / F$ & 48 & Dieta & --- & --- & --- & $\rightarrow$ & $\rightarrow$ & --- & $\rightarrow$ & $\rightarrow$ \\
\hline & 47 a 46 & 30 a 64 & $M / F$ & 48 & Controle & -- & -- & --- & $\rightarrow$ & $\rightarrow$ & --- & $\rightarrow$ & $\rightarrow$ \\
\hline \multirow{4}{*}{$\begin{array}{l}\text { Varady et } \\
\text { al. }(2007)^{12}\end{array}$} & 18 & 40 a 70 & $\mathrm{~F} / \mathrm{M}$ & 8 & Step/ Ciclo & 3 & $25-40$ & $\begin{array}{l}60-75 \% \\
\text { FCmáx }\end{array}$ & $\rightarrow$ & $\rightarrow$ & --- & $\uparrow$ & $\downarrow$ \\
\hline & 18 & 40 a 70 & $\mathrm{~F} / \mathrm{M}$ & 8 & $\begin{array}{l}\text { Step/ Ciclo + } \\
\text { esterol vegetal }\end{array}$ & 3 & $25-40$ & $\begin{array}{l}\text { 60-75\% } \\
\text { FCmáx }\end{array}$ & $\downarrow$ & $\downarrow$ & --- & $\uparrow$ & $\downarrow$ \\
\hline & 18 & 40 a 70 & $\mathrm{~F} / \mathrm{M}$ & 8 & Esterol vegetal & --- & --- & --- & $\downarrow$ & $\downarrow$ & --- & --- & --- \\
\hline & 20 & 40 a 70 & $\mathrm{~F} / \mathrm{M}$ & 8 & Controle & --- & --- & --- & $\rightarrow$ & $\rightarrow$ & --- & $\rightarrow$ & $\rightarrow$ \\
\hline
\end{tabular}

$\mathrm{CT}=$ colesterol total; $\mathrm{LDL}=$ lipoproteína de baixa densidade; $\mathrm{VLDL}=$ lipoproteína de densidade muito baixa; HDL= lipoproteína de alta densidade; $\mathrm{TG}=$ triglicérides; $M=$ masculino; $F=$ feminino; $\mathrm{VO}_{2} \max =$ consumo máximo de oxigênio; $F C$ max= frequência cardíaca máxima; $\downarrow=$ reduz; $\rightarrow=$ mantém; $\uparrow=$ aumenta; $---=$ não relatado.

o exercício realizado em cicloergômetro. O efeito da supervisão do treinamento aeróbico foi comparado em dois estudos e se mostrou mais efetivo na presença de supervisão.

Os resultados dos estudos que analisaram o treinamento aeróbico indicaram que o colesterol total foi reduzido em um estudo; o LDL reduziu em dois estudos; o VLDL e os TG reduziram em cinco estudos; e o HDL aumentou em 10 estudos. Quando o exercício físico foi associado às intervenções dietéticas ou medicamentosas as reduções no colesterol total, LDL e triglicérides foram mais acentuadas. Todavia, nenhum efeito adicional foi verificado para os níveis de HDL colesterol com tais associações.

Ao analisar a caracterização do treinamento aeróbico nestes desfechos pode ser observado que a modificação favorável no perfil lipídico é evidenciada com os exercícios realizados em cicloergômetro, caminhada ou corrida, com frequência semanal de 3-5vezes, duração de 25-60 min/sessão e intensidade entre 60-75\%FCmax, 10\% abaixo do limiar anaeróbio ou quando foi totalizado um volume semanal equivalente a 16 milhas. Em especial, para a redução do LDL, bem como para o aumento do HDL, verificou-se que o protocolo de treinamento, eventualmente, requer uma duração ainda maior da sessão, ao redor de $120 \mathrm{~min}$.

Os estudos em que foi avaliado o efeito do treinamento de força nas lipoproteínas estão apresentados na tabela 4. 
Tabela 4 - Efeito crônico do exercício de força no perfil lipídico de seus praticantes.

\begin{tabular}{|c|c|c|c|c|c|c|c|c|c|c|c|c|c|c|c|c|}
\hline & & Dados & Demogra & áficos & Caracteriz & ação do & Treinam & iento & & & & Efei & & & & \\
\hline $\begin{array}{l}\text { Autor } \\
\text { (ano) }\end{array}$ & $N$ & Pop. & Idade & Gênero & Exposição & $\begin{array}{l}\text { Freq. } \\
(x / \text { sem })\end{array}$ & Séries & Rep. & Intensidade & $\begin{array}{l}\text { Intervalo } \\
\text { entre as } \\
\text { séries (seg) }\end{array}$ & $\begin{array}{l}\text { Exercícios } \\
\text { (número) }\end{array}$ & $\mathrm{CT}$ & LDL & VLDL & $\mathrm{HDL}$ & TG \\
\hline \multirow{3}{*}{$\begin{array}{l}\text { Polito et al. } \\
(2010)^{16}\end{array}$} & 7 & & $29 \pm 1$ & & & 3 & 2 & $10-20$ & $\begin{array}{l}\text { Próximo à } \\
\text { fadiga }\end{array}$ & $50-180$ & 10 & --- & --- & --- & --- & $\rightarrow$ \\
\hline & & NDLP & & $M$ & 12 & & & & & & & & & & & \\
\hline & $7 \mathrm{CO}$ & & $27 \pm 1$ & & & & & & & & & --- & --- & --- & --- & --- \\
\hline \multirow{3}{*}{$\begin{array}{l}\text { Boyden et } \\
\text { al. }(1993)^{14}\end{array}$} & 46 & & & & & 3 & 3 & 8 & $70 \% 1 \mathrm{RM}$ & --- & 12 & $\downarrow$ & $\downarrow$ & --- & $\rightarrow$ & $\rightarrow$ \\
\hline & & NDLP & 28 a 39 & $\mathrm{~F}$ & 20 & & & & & & & & & & & \\
\hline & $42 \mathrm{CO}$ & & & & & & & & & & & --- & --- & --- & --- & -- \\
\hline \multirow{3}{*}{$\begin{array}{l}\text { Gavin et al. } \\
(2010)^{14}\end{array}$} & 64 & & & & & 3 & $2-3$ & 8 & --- & --- & 12 & $\rightarrow$ & $\rightarrow$ & $\downarrow$ & $\rightarrow$ & $\downarrow$ \\
\hline & & DLP & $55 \pm 8$ & $F / M$ & 24 & & & & & & & & & & & \\
\hline & $\begin{array}{l}63 \\
\mathrm{CO}\end{array}$ & & & & & & & & & & & -.- & & --- & --- & \\
\hline
\end{tabular}

Pop= população; Rep=repetições; CT= Colesterol Total; LDL= Lipoproteína de baixa densidade; VLDL= Lipoproteína de muito baixa densidade; $\mathrm{HDL}=$ lipoproteína de alta densidade; $\mathrm{TG}=$ triglicérides; $\mathrm{M}=$ masculino; $\mathrm{F}=$ feminino; $\mathrm{NDLP}=$ Normolipidêmico; $\mathrm{DLP}=\mathrm{Dislipidêmico;} C \mathrm{CO}$ controle; $1 \mathrm{RM}=1$ repetição máxima; $\downarrow=$ reduz; $\rightarrow=$ mantém; $\uparrow=$ aumenta; ---= não relatado.

Como pode ser verificado, nenhum estudo realizou o treinamento de força em indivíduos dislipidêmicos e, somente em um deles, indivíduos do gênero feminino foram incluídos na amostra. A idade dos sujeitos variou de 28 a 70 anos e nenhum estudo relatou o uso de medicamentos hipolipemiantes ou considerou qualquer controle dietético durante a sua realização. A duração do treinamento de força foi de 12 a 24 semanas, e frequência de 3 sessões/sem, compostas de 10-12 exercícios para membros superiores e inferiores. Foram executados 8-20 repetições/exercício com intensidade média de $70 \%$ de 1 repetição máxima (RM) ou próximo da fadiga concêntrica.

Reduções do colesterol total, das LDL, das VLDL e dos TG com o treinamento de força foram verificadas em apenas um estudo, ao passo que nenhuma alteração nas HDL foi identificada. Assim, a caracterização do treinamento de força para reduzir o colesterol total e o LDL foi composta por treinamento de força com 20 semanas de duração, realizados 3sessões/sem, em três séries com oito repetições em uma intensidade de 70\%1RM. Já, para a redução das VLDLs e dos TG, o treinamento de força foi realizado por 24 semanas, 3sessões/sem, conduzidos em 2-3 séries com oito repetições cada. No entanto, a intensidade ideal para este fim não foi relatada.

\section{DISCUSSÃO}

Os principais achados desta revisão sistemática advogam em favor da realização dos exercícios aeróbicos, tanto aguda quanto cronicamente, para a redução dos níveis de TG e elevação dos níveis de HDL. Além disso, foi observado que há carências de investigações bem controladas sobre os efeitos dos exercícios de força no controle lipêmico.

A síntese dos estudos que analisaram o efeito do treinamento aeróbico sobre os níveis de colesterol e triglicérides mostra que este tipo de intervenção reduz ou mantém essas variáveis, exceto para o HDL em que foi verificada manutenção ou mesmo elevação. Esses achados ratificam o treinamento aeróbico como terapia não medicamentosa eficiente no tratamento das alterações das dislipidemias.

Tem sido relatado na literatura que o treinamento aeróbico é mais efetivo nos indivíduos que apresentam níveis dos lípides sanguíneos alterados ${ }^{9,10}$. No entanto, ao analisar somente os estudos com amostra de sujeitos dislipidêmicos, verifica-se que somente dois ${ }^{11,12}$ mostraram redução dos níveis e somente quando houve asso- 
ciação com fármacos ${ }^{12}$ ou dieta ${ }^{11}$, não sendo constatado efeito isolado do exercício físico. Por outro lado, para o HDL há somente um estudo ${ }^{12}$ que verificou elevação significante na população dislipidêmica, de modo que o protocolo de treinamento a ser seguido deve considerar exercícios realizados no cicloergômetro ou "step" por, pelo menos, oito semanas e realizado em 3 sessões/sem, com $25-40 \mathrm{~min} / \mathrm{sessão} \mathrm{em}$ intensidade de $60-75 \%$ FCmax. Por fim, ao se almejar reduzir os níveis de triglicérides com o treinamento aeróbico em sujeitos dislipidêmicos, verifica-se que há apenas dois estudos ${ }^{12,13}$ na literatura, e o protocolo de treinamento destes estudos foram constituídos por exercícios realizados em cicloergômetros, "step" ou corrida por um tempo de 8-9 semanas, realizados 3vezes/sem, com 25-50 min/sessão e numa intensidade de $75 \% \mathrm{VO}_{2}$ max. Diante do exposto, vale lembrar que a IV Diretriz Brasileira sobre Dislipidemias e Prevenção da Aterosclerose ${ }^{5}$ recomenda que os exercícios aeróbicos devem ser realizados de 3-6vezes/sem, em sessões de 30-60 min, em intensidade de 60-80\% FCmax. Assim, este estudo pôde agregar à recomendação quanto ao tempo mínimo de oito semanas de exposição ao treinamento aeróbico para que o sujeito com dislipidemia seja beneficiado com alterações favoráveis nos marcadores da doença (TG, LDL e HDL).

O corpo de evidências científicas referentes aos efeitos do treinamento aeróbico nos lípides é bem maior em indivíduos normolipêmicos. De modo geral, a recomendação da IV diretriz brasileira sobre dislipidemias e prevenção da aterosclerose ${ }^{5}$ contempla as características dos exercícios que tiveram efeitos favoráveis nos níveis circulantes dos lípides sanguíneos. Porém, mais uma vez vale ressaltar a necessidade de se agregar à recomendação disponível o tempo mínimo de exposição a que os sujeitos devem permanecer em treinamento. $\mathrm{Na}$ presente revisão, verificou-se que o tempo minimamente efetivo foi de 12 semanas de treinamento físico.

$\mathrm{Na}$ IV Diretriz Brasileira sobre Dislipidemias e Prevenção da Aterosclerose ${ }^{5}$ há também a recomendação do treinamento de força com imposição de sobrecargas de 50\% de 1RM. Nesta revisão verificamos que o corpo de evidências é composto por apenas três estudos ${ }^{14-16}$, sendo que somente em um deles foram estudados sujeitos com dislipidemias ${ }^{15}$. Neste estudo o desfecho verificado com o treinamento de força foi à redução nos níveis de TG, empregando um protocolo de treinamento que consistiu de 24 semanas de exercícios seriados (2-3 séries) com oito repetições em um total de 12 exercícios. Porém, nenhum relato foi feito quanto à intensidade do esforço realizado para cada exercício. Assim, o efeito do treinamento de força para sujeitos dislipidêmicos ainda precisa ser mais estudado. Com relação aos estudos em normolipêmicos, o corpo de evidências disponíveis também é reduzido ${ }^{15,16}$. Entretanto, o treinamento de força parece ser efetivo em reduzir os níveis de colesterol total e de LDL quando contemplado um tempo mínimo de 20 semanas de treinamento físico, 3 sessões/sem, em um total de 12 exercícios, sendo executados 8 repetições/série, na intensidade de 70\%1RM.

A análise do efeito agudo do exercício torna-se importante na medida em que o aumento da demanda metabólica associada às contrações musculares provoca diversos ajustes que auxiliam na depuração rápida das lipoproteínas de densidades reduzidas $^{17}$ (quilomícron, VLDL, IDL e LDL), diminuindo a suscetibilidade de oxidação lipídica e aumentando a sobrevida da HDL. Na presente revisão sistemática, verificou-se que este interesse tem sido avaliado somente como medida profilática e após a execução do exercício aeróbico. Porém, mesmo assim, é importante mencionar que são poucos os trabalhos que avaliaram os efeitos agudos do exercício aeróbico ${ }^{18-22}$. Os resultados indicaram que a redução dos níveis sanguíneos de TG após uma única realização de exercícios aeróbicos deve contemplar a realização 
de exercícios em cicloergômetro ou esteira, por 30-90min/sessão e intensidade de 50-60\% $\mathrm{VO}_{2}$ max. Já para a elevação dos níveis de HDL, as características a serem consideradas são: realização dos exercícios na esteira, por $90 \mathrm{~min} /$ sessão e intensidade de $50-75 \% \mathrm{VO}_{2}$ max. Confrontando os protocolos de exercícios para prover alterações benéficas nos níveis circulantes de TG e HDL verifica-se que eles se diferenciam no tipo de exercício realizado, uma vez que para os TG, exercícios realizados tanto no cicloergômetro quanto na esteira resultaram em reduções significantes, ao passo que a elevação do HDL somente foi verificada após os exercícios realizados em esteira. Isso remete que possivelmente o tamanho da massa muscular exercitada seja um aspecto importante a ser considerado na formulação do protocolo de exercícios aeróbicos. De fato, quanto maior a massa muscular exercitada, maior o fluxo sanguíneo destinado para atender a maior demanda metabólica total, ou seja, para atender ao maior gasto calórico do exercício. Assim, muito provavelmente, é por este motivo que no estudo de Gordon et al. ${ }^{19}$ foi verificado elevação significante do HDL, pois em seu protocolo de exercícios era solicitado que os sujeitos atingissem um gasto calórico de aproximadamente $800 \mathrm{kcal}$.

O presente estudo apresenta algumas limitações que devem ser consideradas. $\mathrm{O}$ método de pesquisa de artigos limitou-se em avaliar os estudos publicados em periódicos indexados nas bases de dados eletrônicas PubMed/MedLine, LILACS e SCIELO. Dessa forma, é possível que alguns estudos sobre esta temática não tenham sido incluídos. Contudo, vale ressaltar que as bases de dados utilizadas no presente estudo são as mais consultadas para pesquisa bibliográfica de manuscritos em português e inglês. Ademais, a pesquisa bibliográfica desse estudo não incluiu bancos de dados de teses e dissertações, o que certamente impactou em um menor número de estudos analisados. A pesquisa bibliográfica foi realizada utilizando apenas descritores em português ou inglês e estudos existentes em outros idiomas não foram incluídos.

Diante do exposto, é possível concluir que há um corpo de evidências reduzido referente ao exercício físico como medida terapêutica das dislipidemias e que a recomendação de exercícios da IV Diretriz Brasileira sobre Dislipidemias e Prevenção da Aterosclerose ${ }^{5}$ contempla grande parte das características do treinamento aeróbico que tem se mostrado efetivo para melhoria das lípides plasmáticas, exceto para o tempo mínimo de oito semanas de realização deste treinamento. Já para o treinamento de força, a evidência que se tem é sustentada por um único estudo que observou que o tempo mínimo de treinamento deve ser de 24 semanas, em uma frequência de 2-3 sessões por semana, com oito repetições em um total de 12 exercícios. Não há, até o momento, evidências de efeitos agudos do treinamento de força para o tratamento de dislipidêmicos.

\section{Contribuição dos autores}

Allan Irwin Leite Bezerra- aluno de graduação, utilizou o trabalho para a conclusão do curso (monografia) e como pesquisador 01 realizou a busca e seleção dos artigos, tabulou e analisou os dados; Hélcio Kanegusuku aluno de pós-graduação (doutorado) foi o pesquisador 02 e realizou a busca e seleção dos artigos, tabulou e analisou os dados; Wagner Luiz do Prado (Doutor com vasta experiência em lípides) foi o revisor do artigo e contribuiu na discussão do mesmo. Raphael Mendes Ritti Dias (Doutor com vasta experiência em aterosclerose periférica) foi o revisor do artigo e contribuiu na metodologia e discussão do mesmo; Crivaldo Gomes Cardoso Júnior (Doutor) foi o mentor intelectual do artigo, orientou o estudo e conduziu a análise e discussão dos dados. 


\section{REFERÊNCIAS}

1. Third Report of the National Cholesterol Education Program (NCEP) Expert Panel on Detection, Evaluation, and Treatment of High Blood Cholesterol in Adults (Adult Treatment Panel III) final report. Circulation 2002;106:3143-3421.

2. Obesity: preventing and managing the global epidemic. Report of a WHO consultation. World Health Organ Tech Rep Ser 2000;894:i-xii, 1-253.

3. Gigante DP,Moura EC and Sardinha LM. Prevalence of overweight and obesity and associated factors, Brazil, 2006. Rev Saude Publica 2009;43 Suppl 2:83-89.

4. Boden WE. High-density lipoprotein cholesterol as an independent risk factor in cardiovascular disease: assessing the data from Framingham to the Veterans Affairs High-Density Lipoprotein Intervention Trial. Am J Cardiol 2000;86:19L-22L.

5. Sposito ACC, B.; Fonseca, F.A.H.; Bertolami, M.C. IV DIRETRIZ BRASILEIRA SOBRE DISLIPIDEMIAS E PREVENÇÃO DA ATEROSCLEROSE (IVDBDPA) DEPARTAMENTO DE ATEROSCLEROSE DA SOCIEDADE BRAILEIRA DE CARDIOLOGIA. Arquivos Brasileiros de Cardiologia 2007;88:2-19.

6. Morris JN, Kagan A, Pattison DC and Gardner MJ. Incidence and prediction of ischaemic heart-disease in London busmen. Lancet 1966;2:553-559.

7. Halbert JA, Silagy CA, Finucane P, Withers RT and Hamdorf PA. Exercise training and blood lipids in hyperlipidemic and normolipidemic adults: a meta-analysis of randomized, controlled trials. Eur J Clin Nutr 1999;53:514-522.

8. Maher CG, Sherrington C, Herbert RD, Moseley AM and Elkins M. Reliability of the PEDro scale for rating quality of randomized controlled trials. Phys Ther 2003;83:713-721.

9. Couillard C, Despres JP, Lamarche B, Bergeron J, Gagnon J, Leon AS, Rao DC, Skinner JS, Wilmore JH and Bouchard C. Effects of endurance exercise training on plasma HDL cholesterol levels depend on levels of triglycerides: evidence from men of the Health, Risk Factors, Exercise Training and Genetics (HERITAGE) Family Study. Arterioscler Thromb Vasc Biol 2001;21:1226-1232.

10. Tran ZV, Weltman A, Glass GV and Mood DP. The effects of exercise on blood lipids and lipoproteins: a meta-analysis of studies. Med Sci Sports Exerc 1983;15:393-402.

11. Stefanick ML, Mackey S, Sheehan M, Ellsworth N, Haskell WL and Wood PD. Effects of diet and exercise in men and postmenopausal women with low levels of HDL cholesterol and high levels of LDL cholesterol. N Engl J Med 1998;339:12-20.

12. Varady KA, Houweling AH and Jones PJ. Effect of plant sterols and exercise training on cholesterol absorption and synthesis in previously sedentary hypercholesterolemic subjects. Transl Res 2007;149:22-30.

13. Raz I, Rosenblit H and Kark JD. Effect of moderate exercise on serum lipids in young men with low high density lipoprotein cholesterol. Arteriosclerosis 1988;8:245-251.

14. Boyden TW, Pamenter RW, Going SB, Lohman TG, Hall MC, Houtkooper LB, Bunt JC, Ritenbaugh $\mathrm{C}$ and Aickin M. Resistance exercise training is associated with decreases in serum low-density lipoprotein cholesterol levels in premenopausal women. Arch Intern Med 1993;153:97-100.

15. Gavin C, Sigal RJ, Cousins M, Menard ML, Atkinson M, Khandwala F, Kenny GP, Proctor $\mathrm{S}$ and Ooi TC. Resistance exercise but not aerobic exercise lowers remnant-like lipoprotein particle cholesterol in type 2 diabetes: a randomized controlled trial. Atherosclerosis 2010;213:552-557.

16. Polito MDCESG, M.A.; Januário, R.S.B. 12-week resistance training effect on muscular strengh, body composition and triglycerides in sedentary man. . Revista Brasileira de Medicina do Esporte 2010;16:29-32.

17. Ficker ES, Maranhao RC, Chacra AP, Neves VC, Negrao CE, Martins VC and Vinagre CG. Exercise training accelerates the removal from plasma of LDL-like nanoemulsion in moderately hypercholesterolemic subjects. Atherosclerosis 2010;212:230-236.

18. Gill JM, Al-Mamari A, Ferrell WR, Cleland SJ, Sattar N, Packard CJ, Petrie JR and Caslake MJ. Effects of a moderate exercise session on postprandial lipoproteins, apolipoproteins and lipoprotein remnants in middle-aged men. Atherosclerosis 2006;185:87-96.

19. Gordon PM, Visich PS, Goss FL, Fowler S, Warty V, Denys BJ, Metz KF and Robertson J. Comparison of exercise and normal variability on HDL cholesterol concentrations and lipolytic activity. Int J Sports Med 1996;17:332-337. 
20. Mitchell JB, Rowe JR, Shah M, Barbee JJ, Watkins AM, Stephens C and Simmons S. Effect of prior exercise on postprandial triglycerides in overweight young women after ingesting a high-carbohydrate meal. Int J Sport Nutr Exerc Metab 2008;18:49-65.

21. Miyashita M and Tokuyama K. Moderate exercise reduces serum triacylglycerol concentrations but does not affect pre-heparin lipoprotein lipase concentrations after a moderate-fat meal in young men. Br J Nutr 2008;99:1076-1082.

22. Schlierf G, Dinsenbacher A, Voggenreiter U, Drews B, Kather H and Kohlmeier M. Plasmatriglycerides and exercise: a delicate balance. Klin Wochenschr 1988;66:129-133.

23. Alam S, Stolinski M, Pentecost C, Boroujerdi MA, Jones RH, Sonksen PH and Umpleby AM. The effect of a six-month exercise program on very low-density lipoprotein apolipoprotein $\mathrm{B}$ secretion in type 2 diabetes. J Clin Endocrinol Metab 2004;89:688-694.

24. Agren B, Olin C, Castenfors J and Nilsson-Ehle P. Improvements of the lipoprotein profile after coronary bypass surgery: additional effects of an exercise training program. Eur Heart J 1989;10:451-458.

25. Brown AJ, Setji TL, Sanders LL, Lowry KP, Otvos JD, Kraus WE and Svetkey PL. Effects of exercise on lipoprotein particles in women with polycystic ovary syndrome. Med Sci Sports Exerc 2009;41:497-504.

26. Busby J, Notelovitz M, Putney K and Grow T. Exercise, high-density lipoprotein-cholesterol, and cardiorespiratory function in climacteric women. South Med J 1985;78:769-773.

27. Cauley JA, Kriska AM, LaPorte RE, Sandler RB and Pambianco G. A two year randomized exercise trial in older women: effects on HDL-cholesterol. Atherosclerosis 1987;66:247-258.

28. Cox KL, Puddey IB, Morton AR, Masarei JR, Vandongen R and Beilin LJ. Controlled comparison of effects of exercise and alcohol on blood pressure and serum high density lipoprotein cholesterol in sedentary males. Clin Exp Pharmacol Physiol 1990;17:251-255.

29. Grandjean PW, Oden GL, Crouse SF, Brown JA and Green JS. Lipid and lipoprotein changes in women following 6 months of exercise training in a worksite fitness program. J Sports Med Phys Fitness 1996;36:54-59.

30. Marti B, Suter E, Riesen WF, Tschopp A, Wanner HU and Gutzwiller F. Effects of long-term, self-monitored exercise on the serum lipoprotein and apolipoprotein profile in middle-aged men. Atherosclerosis 1990;81:19-31.

31. Moreira MM, Souza HP, Schwingel PA, Sa CK and Zoppi CC. Effects of aerobic and anaerobic exercise on cardiac risk variables in overweight adults. Arq Bras Cardiol 2008;91:200-206, 219-226.

32. Nieman DC, Brock DW, Butterworth D, Utter AC and Nieman CC. Reducing diet and/or exercise training decreases the lipid and lipoprotein risk factors of moderately obese women. J Am Coll Nutr 2002;21:344-350.

33. Parente EB, Guazzelli I, Ribeiro MM, Silva AG, Halpern A and Villares SM. [Obese children lipid profile: effects of hypocaloric diet and aerobic physical exercise]. Arq Bras Endocrinol Metabol 2006;50:499-504.

34. Stein RA, Michielli DW, Glantz MD, Sardy H, Cohen A, Goldberg N and Brown CD. Effects of different exercise training intensities on lipoprotein cholesterol fractions in healthy middleaged men. Am Heart J 1990;119:277-283.

35. Stolinski M, Alam S, Jackson NC, Shojaee-Moradie F, Pentecost C, Jefferson W, Christ ER, Jones RH and Umpleby AM. Effect of 6-month supervised exercise on low-density lipoprotein apolipoprotein B kinetics in patients with type 2 diabetes mellitus. Metabolism 2008;57:16081614.

36. Williams PT, Krauss RM, Vranizan KM, Albers JJ, Terry RB and Wood PD. Effects of exerciseinduced weight loss on low density lipoprotein subfractions in healthy men. Arteriosclerosis 1989;9:623-632.

37. Williams PT, Krauss RM, Vranizan KM and Wood PD. Changes in lipoprotein subfractions during diet-induced and exercise-induced weight loss in moderately overweight men. Circulation 1990;81:1293-1304.

38. Williams PT, Albers JJ, Krauss RM and Wood PD. Associations of lecithin: cholesterol acyltransferase (LCAT) mass concentrations with exercise, weight loss, and plasma lipoprotein subfraction concentrations in men. Atherosclerosis 1990;82:53-58.

39. Williams PT, Stefanick ML, Vranizan KM and Wood PD. The effects of weight loss by exercise or by dieting on plasma high-density lipoprotein (HDL) levels in men with low, intermediate, 
and normal-to-high HDL at baseline. Metabolism 1994;43:917-924.

40. Wood PD, Haskell WL, Blair SN, Williams PT, Krauss RM, Lindgren FT, Albers JJ, Ho PH and Farquhar JW. Increased exercise level and plasma lipoprotein concentrations: a one-year, randomized, controlled study in sedentary, middle-aged men. Metabolism 1983;32:31-39.

41. Holme I, Hostmark AT and Anderssen SA. ApoB but not LDL-cholesterol is reduced by exercise training in overweight healthy men. Results from the 1-year randomized Oslo Diet and Exercise Study. J Intern Med 2007;262:235-243.

Endereço para Correspondência Crivaldo Gomes Cardoso Junior Rod. Celso Garcia Cid, km 380 - Campus Universitário - Cx Postal 6001 Londrina - PR CEP 86051-99 Fone: (43) 3371-4228 Fax: (43) 3371-4144 E-mail: crivaldo@gmail.com
Recebido 25/09/2012

Revisado 12/07/2012

Aprovado 28/01/2013 